\title{
Lost in transition-Newly qualified registered nurses and their transition to practice journey in the first six months: A qualitative descriptive study
}

\author{
Yen Tjuin Eugene Teoh, Yi Jia Lim \\ Tan Tock Seng Hospital, Singapore, Singapore
}

Received: February 3, 2019

DOI: $10.5430 /$ jnep.v9n7p38

\author{
Accepted: March 11, 2019 \\ Online Published: March 25, 2019 \\ URL: https://doi.org/10.5430/jnep.v9n7p38
}

\begin{abstract}
Background and objective: Poor transition to practice during the first year of graduate nurses' employment causes compromised patient care and a high staff turnover. Globally, as the world grapples with an ageing population and increasing healthcare demands, it is imperative to retain newly qualified Registered Nurses to sustain the nursing workforce. Objectives: To explore newly qualified Registered Nurses on their transition to practice journey during their first six months of employment.

Methods: Design: A qualitative descriptive design. Settings: A large metropolitan public hospital in Singapore. Participants: A purposive sample of eleven newly qualified Registered Nurses with six months of graduate working experience. Methods: One-to-one semi-structured interviews were conducted, audio-taped and transcribed verbatim. Thematic analysis was employed in this study.

Results: Three themes emerged from the data analysis: personal adaptations, professional adaptations and organisational adaptations. Subthemes of personal adaptations include a new experience, seeking help and coping with transition. Under professional adaptations, the subthemes are accountability, coordination, interprofessional relationships and knowledge. Subthemes of organisational adaptations are staff support, working environment and transition to practice programme.

Conclusions: The findings emphasized the importance of establishing responsibilities, performance expectations and appropriate workplace behaviors guidelines, and to bridge the gap between transition to practice programme and preceptorship is also necessary.
\end{abstract}

Key Words: Graduate, Nurses, Orientation, Qualitative research

\section{INTRODUCTION}

The transition from a neophyte to an experienced Registered Nurse (RN) is stressful for newly qualified Registered Nurses (NQRNs) being simultaneously challenged with the need to draw on their new knowledge and responsibilities, within the context of dynamic social relationships in the workplace, while adjusting to the new role of being a practising pro- fessional. ${ }^{[1]}$ With increasing healthcare demands, ageing workforces coupled with shortage of nursing professionals, a fast and successful transition to practice (TTP) is a concern for the healthcare organisations and patients. ${ }^{[2]}$ In Singapore as elsewhere, NQRNs are expected to deliver safe, competent patient care while orientating and navigating in a new environment as well as making the necessary role adjustments.

${ }^{*}$ Correspondence: Yen Tjuin Eugene Teoh; Email: Eugene_Yt_Teoh@ttsh.com.sg; Address: Tan Tock Seng Hospital, 11 Jalan Tan Tock Seng, Singapore 308433, Singapore. 
Within the context of Singapore, little is known about the TTP journey of NQRNs as there is a paucity of empirical research in this area.

\subsection{Literature review}

Assimilation of NQRNs into a ward context is a recurring concern both regionally in Asia and internationally that is well documented in many studies. ${ }^{[2-5]}$ This transition journey requires that $\mathrm{NQRNs}$ understand and adapt to both the ward culture ${ }^{[6]}$ and to the hierarchical structure. ${ }^{[4,6,7]}$ Failure to conform to these social rules has been documented to lead to reprimands and hostility from the host staff of the organisation concerned. ${ }^{[2,6]}$

Studies have shown NQRNs report nursing knowledge and clinical skills deficits ${ }^{[2,8-10]}$ during their first year of practice, these being new clinical skills and applying critical thinking. Additionally, NQRNs may be unfamiliar with routines, ${ }^{[11,12]}$ organisation protocols and policies, ${ }^{[2,9,12,13]}$ thus imposing on their ability to prioritise and manage patient care.

Provision of TTP programme appeared inconsistent in the literature reviewed, with little evidence on the effectiveness of TTP programmes and associated effects on NQRNs' transition. $^{[3,7,9,10]}$

In Singapore, TTP programme is mandatory including learning hospital policies and skills refreshment courses. While TTP programmes are implemented at all public and private hospitals in Singapore, the programmes are currently neither standardised nor monitored by Singapore Nursing Board or Ministry of Health. ${ }^{[1]}$

\subsection{Aims}

This research aimed to explore the perspectives of NQRNs towards their TTP journey, in particular:

How did NQRNs perceive and explain their first year of nursing practice? In respect to TTP, what was perceived as either helpful or challenging for this cohort of newly qualified Registered Nurses?

\section{METHOD}

\subsection{Design}

A qualitative descriptive design was adopted to explore NQRNs' perceptions on their TTP journey.

\subsection{Sample}

A purposive sampling was employed until data saturation with inclusion criteria being i) NQRNs aged twenty-one years and above, ii) with exactly six months of working experience as a Registered Nurse.

Published by Sciedu Press
In total, eleven NQRNs between 23 and 38 years old were recruited, consisting of two male participants and nine female participants. Two of the participants work in the Intensive Care Unit (ICU), one works in the Emergency department (ED) and eight in the surgical and medical general wards. Five of the participants were diploma holders from local polytechnics; six were degree holders, five of whom graduated from a local university and one graduated from an Australian University. One participant had previous nursing experience working as an Enrolled nurse while another graduate was a midcareer switch nurse, who worked in a non-healthcare related industry previously.

After ethical approval was obtained from the university and healthcare organisation concerned, posters were put up on nurses' tea rooms, staff lifts and the organisation's intranet. Due to poor response rate, follow up invitation letters were then sent to NQRNs who met the inclusion criteria. NQRNs were requested to send an e-mail to the research team to expressed their intent to participate.

\subsection{Data collection}

The researcher team developed a semi-structured interview guide from the literature. This guide was further reviewed for content validity on relevance of questions by two independent local Nursing Educators working at the study site who were acknowledged to be experts in NQRNs and transition to practice. A pilot interview of 2 NQRNS was also conducted to test the suitability of the questions to capture the phenomenon and the clarity of the questions. ${ }^{[14]}$ The interview data was not included in this study's analysis.

One-to-one interviews that lasted between twenty and seventy minutes were held in private seminar rooms at the concerned hospital to ensure a quiet environment that was free of distraction. The interviews were audio-taped and transcribed verbatim for analysis. ${ }^{[15]}$ Field notes and observations of the informants' nonverbal behaviour such as facial expressions, postures and gestures were documented. ${ }^{[15]}$

\subsection{Data analysis}

In the initial phase, the transcripts were read repeatedly for the researchers to familiarise with the data and identify manifest content. ${ }^{[16]}$ After data collection ended, the transcripts and analysis that were done simultaneously with the interviews were compiled and reviewed. At this phase, latent content were identified ${ }^{[16]}$ using colour codes in addition to manifest content by the main researcher and one of the researchers independently. Input of analysis from more than one researcher enabled the generation of more insights to the topic as compared to analysis done by sole researcher. ${ }^{[17]}$ Based on the analysis, the two researchers compared notes 
with each other and identified meaning quotes, which were grouped into categories after unanimous decisions were reached within the team for each meaning quotes. The categories were later on classified into three major themes and a number of subthemes inherent within these themes.

\subsection{Ethical considerations}

An ethic approval for this project was obtained from the National Health Group Domain Specific Review Board ethical review. Participants received oral and written information about the study during the recruitment phase. Written informed consents were obtained, which included allowing the interview to be recorded. Participants were informed that participation was voluntary and they could withdraw from the research at any point of time. The participants were reassured that their refusal to participate would not affect their career progression in the institute and participants' identities were kept confidential.

\subsection{Rigor}

In order to ensure trustworthiness of the study, application of principles of pertaining to credibility, dependability, confirmability and transferability was adopted.

\section{RESULTS}

Three substantial themes emerged from the data being: 1) Personal adaptations, 2) Professional adaptations 3) Organisational adaptations. Subthemes of "Personal adaptations" are "a new experience", "seeking help", and "coping with transition". Professional adaptations consist of four subthemes, "accountability", "coordination”, "interprofessional relationship" and "knowledge". Subthemes of Organisational adaptations include "staff support", "working environment" and "transition to practice programme".

\subsection{Personal adaptations}

This theme describes the impact of transition to practice had on NQRNs' personal lives and adjustments made to personal life to meet the work demands. Three subthemes identified were namely "a new experience", "seeking help", and "coping with transition".

\subsection{A new experience}

When participants were asked to describe how they felt to be an RN for the past six months, they expressed difficulties in performing the role of an RN as they were "lost", "still new" and "don't have the experience for work". Taking up the role of RN was described as riding an "emotional rollercoaster", "very stress" and feeling "pressured". Another aspect of new experience was adapting to shift work, which proved to be physically demanding and disruptive to social life.

\subsection{Seeking help}

All the participants testified to seeking help when they met difficulties at work. Strategies included seeking help from a senior colleague, self-study, or accessing support documents on hospital protocols and policies through the hospital intranet. However, NQRNs mentioned several barriers when they needed help. Personality was identified as a barrier while some viewed the act of seeking help as a marker of poor performance:

"...I have this problem of delegation. ... because I don't like telling people what to do. So I won't." (Lynette, General ward)

and,

"... asking for help is like admitting that you cannot cope, and it's not a good thing." (Anne, General ward)

\subsection{Coping with transition}

During the transition to practice journey, NQRNs were daunted by performing such new roles as being patients' advocates, patient educators and coordinators of patient care that they did not experience during student days. Imitating the working styles of senior colleagues was a coping strategy to adapt to these new roles.

A number of participants remarked they could not prioritise their tasks at the clinical setting during the first few months. To ensure better time management, participants planned their nursing care prior to work, such as identifying patients who were in poorer condition, listing down the priorities, and reading up on patients' conditions prior to report passing.

A positive attitude was identified from the data which the participants employed when faced with challenges at work:

“...my preceptor ... said... 'Any verbal abuse or any abuse that had been done to you doesn't kill you right? It only makes you stronger.' So from then onwards ... verbal abused you take it in a light manner." (Kevin, General ward)

The clinical environment was recurrently described as stressful by the participants. To assuage emotional distress, participants shared that they sought religious support and confided with family and colleagues. Several participants felt that getting sufficient rest to recuperate relieves them from stress:

"I try to rest a lot, ... rest my mind... Other than that, not putting too much pressure for myself, telling me to do things at my own pace." (Lynette, General ward) 


\subsection{Professional adaptations}

This theme describes the development of professional role during the transition to practice. The subthemes include "accountability", "coordination", "interprofessional relationship" and "knowledge".

\subsection{Accountability}

Participants in this study remarked feeling stressful as they were accountable of delivering safe and competent patient care. Patient safety was a major concern in respect to accountability, which was reflected in the learning attitude of the participants:

“... you have to study the protocols and make sure you know. And all these things are important ... because it's patients' life at stake..." (Nicole, General ward)

\subsection{Coordination}

Coordinating patient care posed challenges for the participants working in the general ward as they were required to handle between six and twelve patients on day shifts. Additionally, they may need to work with doctors from more than one teams based on patients' diagnosis. This presented another challenge for coordinating patient care because during doctor rounds NQRNs could only attend to one team when two or more teams were present at the same time:

"[I felt] stressed... This one [team] talk to you this thing, this one talk to you another thing... I also cannot remember [the orders]..." (Diane, General ward)

\subsection{Interprofessional relationship}

During their TTP, NQRNs recounted that they faced significant problems with the nurses they closely worked with:

“...So they will... gossip behind me, say something bad about me. Even I don't know until somebody tell me... " (Diane, General ward)

Different working styles among nurses were a challenge to the NQRNs and they had to suit different working styles to different experienced nurses to avoid reprimands:

“... if you don't know the staff ... character, then you do ... [something] she don't like. Then you will kena [Singaporean slang: receive'] not so good [treatment]. ... if you do the right way she will ... be very nice to you and will not make trouble for you, will not scold you..." (Helen, General ward)
Communication with the doctors was another common challenge faced by the participants. Some participants had described the doctors as disrespectful to nurses. The notion of hierarchy in the workplace was illustrated by a number of participants who explained this situation as follows:

"...There are some medical teams that make you feel like you are a slave to them,... some rounds we can actually really participate ... but [for] most of the medical teams ... nurses are just there to help them." (Lymette, General ward)

\subsection{Knowledge}

Many participants working in the general wards felt they were able to perform nursing skills competently after they graduated. However, NQRNs who worked in the ICU felt that academic preparation was inadequate to work in the specialised setting. For example, lessons that introduced the equipments used in the ICU were "touch and go" and were not "teach in depth". Another heterogeneous case was Terry, who completed his degree in Australia. As the focus of the curriculum was on diseases prevalent in Australia, he was not prepared for neurology-related nursing skills which were required in his working unit.

These NQRNs interviewed had to pick up new clinical knowledge, which consisted of understanding institutional protocols, work flow, documentation, and new diagnosis or medicine. However, the learning process was impeded by time constraints:

“... your first priority is more like finish your job quick, quickly and safely. So learning is like it's being pushed down to second." (Terry, General ward)

To overcome this challenge, all the participants remarked that they would read-up at home to improve their clinical knowledge and seized opportunities to practice clinical skills, such as administering intravenous medications. NQRNs in this study found translation of knowledge to practice to be difficult because the complex co morbidity of their patients' conditions. The translation of clinical skills knowledge was difficult too: In the university learning setting, NQRNs needed to follow the steps in the curriculum strictly, which could be time consuming. At work, efficiency took precedence over academic learning; hence the participants commented they needed to be flexible to integrate nursing rationale with work efficiency:

“...experience-wise and theory-wise is a totally different thing ... if you combine both of them ... you better rationalise things, as in why they 
do it in certain ways and why some things you re-

ally cannot cut short, so that patient's life would not jeopardized." (Kevin, General ward)

\subsection{Organizational adaptations}

This theme described organisational factors that influenced NQRNs' transition as they assimilated into the organisational culture and identified themselves as a member of their institute of employment. Three subthemes identified were "staff support", "working environment" and "TTP programme".

\subsection{Staff support}

The participants identified emotional support from nursing staff and nursing management such as a welcoming attitude towards the NQRNs, encouragement and forgiving towards NQRNs' incompetence due to their inexperience. Support to facilitate learning was also crucial, such as providing preceptors, guidance provided by fellow staff, and receiving feedback from nurse managers. However, the learning process was challenged by manpower limitations that resulted in lack of preceptor. Ad hoc guidance was not readily available when other staff members were busy or colleagues were unwilling to provide guidance. The following accounts described these situations:

“... she doesn't have the patience to teach. She's not so approachable ... So even though she has the knowledge and the seniority, I don't think she is a good preceptor..." (Anne, General ward),

and;

“... I keep following different people and different staff have different styles... which is sometimes... contraindicated lah. [Singaporean slang: indicating tone']." (Sue, ICU)

Aside from support in the working unit, a buddy system was initiated by the institution of this study to provide NQRNs with work advice and emotional support. However, the feedback for the buddy system was negative:

"They should have a protected time for those buddies. ... You should assimilate them together with the newbies. If not, it's like a name; it doesn't work as well as what it's supposed to be." (Kevin, General ward)

and;

“... my department [is a] more specialized department. ... I've never contacted my buddy. . .
Because in my opinion, if you are not from the same working environment I don't think you would understand what troubles I am going through." (Abby, ED)

\subsection{Working environment}

Familiarity with the clinical area, workloads and administrative duties were the main factors that influenced the transition. Four participants mentioned that they were posted back to the discipline where they had their final placement during their academic days. This allowed a smoother transition as they were familiar with the environment and routines. On the other hand, a high administrative workload compounded time constraints:

"...there's a lot of paper work and at the same time your computerised updating system and at the same time you have to pick [action of drawing medication from the automated dispensing cabinet] your own medication for the next shift..." (Terry, General ward)

The considerable amount of time spent on assigned workload resulted in work dissatisfaction as some NQRNs felt "mechanical as a worker". Some participants lamented that patient communication time was sacrificed to complete daily workload.

\subsection{Transition to practice programme}

Although there was a general consensus that TTP programmes helped to standardise nursing practices, those who worked in specialised units felt that the unit specific orientation programme was more relevant for them and was able to cover discipline-specific nursing skills:

"But sometimes the hospital orientation... are not applicable to ICU setting. Like they keep saying general ward you should do this you should do that...I prefer the unit based orientation." (Sue, ICU)

\section{Discussion}

The recruited NQRNs in the current study testified to feeling immense pressure during their transition year. They felt lost and were challenged to perform different roles of an RN. However, few sought help as they feared being seen as incompetent. Similar findings were reported in international literature in which participants described as not wanting to appear "stupid". ${ }^{[2,5]}$ The findings suggest role ambiguity and that the NQRNs' had high expectations on themselves to perform on a par as a seasoned RN. Therefore, job descriptions regarding NQRNs' responsibilities, autonomy, work 
demands and performance expectations should be generated to reduce stress resulting from these issues.

Participants commented that they were accountable for all nursing decisions made in the course of patient care. Implicit in the interviews was the fear of patients' losing their lives if any mistake was made. Such findings were echoed in previous literature that participants revealed feeling burdened as they were responsible for human lives. ${ }^{[9,18]}$

The participants repeatedly mentioned they were new staff, which suggests they perceived themselves at the bottom of the hierarchical structure in the working environment. The participants also experienced acts of horizontal violence such as gossip and discouragement from their colleagues ${ }^{[19]}$ but this was not reported to higher authority.

As Singapore society is heavily influenced by Asian values to display high deference to seniors and authority, ${ }^{[20]} \mathrm{NQRNs}$ might tolerate horizontal violence because they felt they had the lowest position in the ward. Consistent with this findings, horizontal violence from senior nurses and existence of in traprofessional and interprofessional hierarchical structures were reported in previous research. ${ }^{[4,6,10]}$ This suggests horizontal violence and establishing hierarchical structure are common workplace practices. It has been recognized that horizontal violence is emotionally draining and could result in compromised patient care and higher turnover ${ }^{[19]}$ Hence, a culture that condemns horizontal violence should be promoted and acceptable professional conducts and attitudes should be stated to help NQRNs identify acts of horizontal violence.

Concurring with previous regional and international literature, ${ }^{[2,11]}$ knowledge deficit was identified as a transition barrier. The novice to expert model by Benner (1982) ${ }^{[21]}$ could in part explain why the NQRNs faced challenges in acquiring and translating knowledge in practice. ${ }^{[10,12]}$ In this model, a neophyte nurse is at the advanced beginner stage where they had prior experience in nursing but is unable to identify crucial information that warrants attention. ${ }^{[12]}$ Hence NQRNs needed more exposure to recognise such meaningful situations which was described as needing "more experience" by the participants in this study. Furthermore, an advanced beginner would rely on work instructions but practice discretionary judgement, which is demonstrated by NQRNs in this study as justifying workplace practices with scientific rationales. Hence, discussion sessions facilitated by nurse educators could be arranged for NQRNs to share their knowledge and nurse educators could provide guidance on evidence-based nursing care to address knowledge deficit. Staff support was crucial for assimilating NQRNs into or-

Published by Sciedu Press ganisational culture and facilitating learning process that develops NQRNs into a contributing team member. ${ }^{[22]}$ Despite positive accounts, lack of supervision and unhelpful colleagues were some barriers identified. These findings are congruent with the literature that describes a dismissive attitude toward $\mathrm{NQRNs}^{[4]}$ and absence of supervision and guidance. ${ }^{[4,23]}$

Of note is that despite having a buddy system being implemented in the hospital under investigation, this was underused by the participants in this study. Buddies are assigned to NQRNs to assist them in their social assimilation to the organisation's culture during their initial phase of the career. Assigned buddies do not have any mentoring or preceptoring responsibilities. Participants chose to adopt a positive attitude to work or referred to family and friends when they were stress. The buddy system did not fulfill its intent of providing support to NQRNs for work advice. Inadequate interaction to build rapport was a barrier identified. Another barrier was difference in working units between NQRNs and their buddies because the participants believed they would not share the same experiences as their buddies if the buddies were from another discipline, leading to reluctance to approach them for advice. Hence, allocating buddies from same disciplines and arranging meeting sessions for NQRNs and buddies may promote the use of the this system.

The working environment proved influential to the TTP for NQRNs for example when overwhelmed with the heavy workload, patient care was task-focused and few participants mentioned the need to address emotional and social issues. This is consistent with the findings found by McKenna, Smith, Poole, and Coverdale (2003): ${ }^{[24]}$ NQRNs focused on mastering routines and technical skills of the job during the first six months and rarely provided needs beyond physical care.

In this study, all participants testified to having attended a one-month TTP programme which introduced hospital policies and protocols and provided skills training. Upon completion of the programme, NQRNs returned to their working unit and were allocated preceptors by nurse managers. However, limited manpower rendered some NQRNs without preceptors or inconsistent supervision. This suggested poor coordination between the TTP programme and preceptor ship offered in the units. Although preceptorship is a component of the TTP programme, it lacked monitoring to ensure all NQRNs received necessary supervision at the units.

\subsection{Limitations}

This study has a few limitations that need to be highlighted. Firstly, the purposive sampling proposed to include NQRNs 
working in specialised and general discipline. However, none of the participants work in the Operating Theatre unit. Hence, findings from this study may not be representative for NQRNs from this discipline.

Secondly, this is a single site study at a public hospital; therefore data generated may not capture the perspectives on transition to practice of NQRNs working in private hospitals. Furthermore, albeit transition to practice programmes are mandatory for NQRNs, the programme structure and content varies among hospitals1. The findings only evaluated the impact of transition to practice programme on transition to practice in the study site. Hence, it may not be generalisable to account for the impact of transition to practice programme on NQRNs' transition to practice journey implemented in other hospitals.

Thirdly, due to time constraints, a one-time interview was conducted to investigate the transition to practice journey in NQRNs with six months of experience. As mentioned before, the transition to practice was found to be most stressful during the first year of employment. Hence, this study may not provide the perceptions of NQRNs towards their transition to practice journey beyond six months of work and findings may not be exhaustive or comparable for NQRNs who had worked for a year.

\subsection{Suggestions for future research}

This study had identified social and organisational factors that influence NQRNs' perspectives of their transition to practice journey. Hence future research should employ an ethnography research design which is advantageous in examining the interaction between individuals and their environment and explaining the far-reaching impact of social dynamics and policies that influence the transition to practice journey for NQRNs. Future research should also seek to evaluate NQRN's perceptions towards the transition to practice among NQRNs with one-year working experience or more because transition is progressive and perceptions may have changed over time. A multi-site study inclusive of public and private hospitals will be beneficial to evaluate transition to practice journey of NQRNs in general.

\section{Conclusion}

This qualitative study we argue has contributed to the understanding of NQRNs' perceptions of their TTP journey in Singapore. Reported was that NQRNs faced role ambiguity and horizontal violence at the workplace. We would recommend that stakeholders should establish job responsibilities, performance expectations and appropriate workplace behaviors to reduce stress induced by these challenges and to promote a culture that condemns horizontal violence. This study also reported that NQRNs had to deliver safe, competent care despite facing knowledge deficit and taking a high patient workload. However, there was a lack of support to facilitate learning and monitoring of their competency. Hence, nurse educators could arrange feedback sessions to monitor NQRNs' learning, while training could be provided to preceptors to train preceptoring skills. Assimilation into the working unit culture was also another challenge identified. Stakeholders should thus look into better integration of NQRNs in to the working unit and optimising the use of institution buddy system to facilitate a smoother transition into the working environment.

\section{ACKNOWLEDGEMENTS}

The authors would like to thank Registered Nurse Pamela Tan for her assistance throughout the study. The authors would also like to extend their appreciation for the funding support received from NHG-HOMER FY12/B01.

\section{CONFlicts of InTEREST Disclosure}

The authors declare that there is no conflict of interest.

\section{REFERENCES}

[1] Teoh YT, Pua LH, Chan MF. Lost in transition-a review of qualitative literature of newly qualified Registered Nurses' experiences in their transition to practice journey. Nurse Education Today. 2013 33(2): 143-147. PMid:23040878 https ://doi.org/10.1016/j nedt. 2012.08.016

[2] Feng RF, Tsai YF. Socialisation of new graduate nurses to practising nurses. Journal of Clinical Nursing. 2012; 21(13-14): 2064-71 PMid:22384849 https://doi.org/10.1111/j.1365-2702.20 $11.03992 . \mathrm{x}$

[3] Goh K, Watt E. From 'dependent on' to 'depended on': The experience of transition from student to registered nurse in a private hospital graduate program. Australian Journal of Advanced Nursing. 2003;
21(1): $14-20$

[4] Kelly J, Ahern K. Preparing nurses for practice: A phenomenological study of the new graduate in Australia. Journal of Clinical Nursing. 2009; 18(6): 910-18. PMid:18665878

[5] Malouf N, West S. Fitting in: A pervasive new graduate nurse need. Nurse Education Today. 2011; 31(5): 488-93. PMid:21036429 https://doi.org/10.1016/j.nedt.2010.10.002

[6] Bisholt BK. The professional socialization of recently graduated nurses-experiences of an introduction program. Nurse Education Today. 2012; 32(3): 278-82. PMid:21546139 https://doi.org/ $10.1016 / \mathrm{j}$. nedt .2011 .04 .001

[7] Newton JM, McKenna L. The transitional journey through the graduate year: A focus group study. International Journal of Nursing 
Studies. 2007; 44(7): 1231-37. PMid:16867302 https://doi.or g/10.1016/j.ijnurstu.2006.05.017

[8] Etheridge SA. Learning to think like a nurse: Stories from new nurse graduates. The Journal of Continuing Education in Nursing. 2007; 38(1): 24-30. https ://doi .org/10.3928/00220124-2007010 $1-05$

[9] Delaney C. Walking a fine line: Graduate nurses' transition experiences during orientation. Journal of Nursing Education. 2003; 42(19): 437-43. PMid:14577729

[10] Dyess SM, Sherman RO. The first year of practice: new graduate nurses' transition and learning needs. The Journal of Continuing Education in Nursing. 2009; 40(9): 403-10. PMid:19754027 https://doi.org/10.3928/00220124-20090824-03

[11] Clark CM, Springer PJ. Nurse residents' first-hand accounts on transition to practice. Nursing Outlook. 2012; 60(4): e2-8. PMid:22000691 https://doi.org/10.1016/j.outlook. 2011.08.003

[12] Ellerton M, Gregor F. A study of transition: the new nurse graduate at 3 months. The Journal of Continuing Education in Nursing. 2003; 34(3): 103. https://doi.org/10.3928/0022-0124-2003050 $1-05$

[13] McKenna LG, Green C. Experiences and learning during a graduate nurse program: An examination using a focus group approach. Nurse Education in Practice. 2004: 4(4): 258-63. PMid:19038167 https://doi.org/10.1016/j.nepr.2004.01.004

[14] Silverman D. Doing qualitative research. 3rd ed. London: SAGE Publications; 2010.

[15] Holloway I, Wheeler S. Qualitative research in nursing and healthcare. 3rd ed. Chichester, United Kingdom: Blackwell; 2010.

[16] Hanson JL, Balmer DF, Giardino AP. Qualitative research methods for medical educators. Academic Pediatrics. 2011; 11(5): 375-86.
PMid:21783450 https://doi.org/10.1016/j.acap.2011.05 .001

[17] Dierckx de Casterlé B, Gastmans C, Bryon E, et al. QUAGOL: A guide for qualitative data analysis. International Journal of Nursing Studies. 2012; 49(3): 360-71.

[18] Mooney M. Facing registration: The expectations and the unexpected. Nurse Education Today. 2007; 27(8): 840-47. PMid:17234304 https://doi.org/10.1016/j.nedt.2006.11.003

[19] Weinand MR. Horizontal violence in nursing: History, impact, and solution. The Journal of Chi Eta Phi Sorority. 2010; 54(1): 23-26.

[20] Lim J, Bogossian F, Ahern K. Stress and coping in Singaporean nurses: A literature review. Nursing and Health Sciences. 2010; 12(2): 251-58. PMid:20602699 https ://doi .org/10.1111/j.1442-2 $018.2010 .00514 . \mathrm{x}$

[21] Benner P. From novice to expert... the Dreyfus model of skill acquisition. American Journal of Nursing. 1982; 82: 402-7. PMid:6917683

[22] Johnstone MJ, Kanitsaki O, Currie T. The nature and implications of support in graduate nurse transition programs: An Australian study. Journal of Professional Nursing. 2008; 24(1): 46-53. PMid:18206843 https://doi.org/10.1016/j.profnurs.2007.06.003

[23] O'Shea M, Kelly B. The lived experiences of newly qualified nurses on clinical placement during the first six months following registration in the Republic of Ireland. Journal of Clinical Nursing. 2007; 16(8): 1534-42. PMid:17655542 https://doi.org/10.1111/j. 1365-2702.2006.01794. $\mathrm{x}$

[24] McKenna BG, Smith NA, Poole SJ, et al. Horizontal violence: Experiences of registered nurses in their first year of practice. Journal of Advanced Nursing. 2003; 42(1): 90-96. https ://doi .org/10.1 $046 / j .1365-2648.2003 .02583 . x$ 